dependent. With the aid of Theorem $\mathrm{V}$ it can be shown that a necessary and sufficient condition for the verification of the hypothesis of Bôcher's theorem is that $M_{k}\left(u_{1}, u_{2}, \ldots, u_{n}\right)$ be of constant rank $m<n$.

NORTHWESTERN UNIVIERSITY, May, 1906.

\title{
SIGNIFICANCE OF THE TERM HYPERCOMPLEX NUMBER.
}

BY PROFESSOR JAMES BYRNIE SHAW.

(Read before the Chicago Section of the American Mathematical Society, April 14, 1906.)

Essentially four definitions of quite different logical import have been given for the term hypercomplex number, or multiple number. The four things so defined differ considerably in their mathematico-philosophical meaning, and while two of them are in a way equivalent, neither of the others can be correlated with these two or with each other as equivalent. It is proposed to examine these four definitions rather critically.

\section{The n-tuple.}

The first definition I shall denominate the Dedekind definition, although Hamilton discussed, many years before, entities defined in the same way. It is of a pure arithmetical character, since it implies only the existence of a set of things we may call numbers, marks, or entities, according as we conceive them to belong to a domain of integrity, an abstract field, or, in general, an aggregate that we can call a range. At first these entities were in a scalar domain, then they were generalized to a rational domain, then to an abstract field, and obviously we may take them from any range. The definition runs substantially thus : *

A set of $n$ ordered marks (entities) $a_{1}, \ldots, a_{n}$ of a field (range) $F$, is called an $n$-tuple element $a$. The symbol $a=$ $\left(a_{1}, \cdots, a_{n}\right)$ employed is purely positional without functional connotation. Its definition implies that $a=b$ if, and only if, $a_{1}=b_{1}, \cdots, a_{n}=b_{n}$.

* Dickson, "O)n hypercomplex number systems"; Transactions Amer. Math. Society, vol. 6 (1905), pp. 344-348. 
This system of $n$-tuple elements is then made to be linear and associative by a set of postulates of such a nature that the sum and the product of any two elements is a third element. Like all arithmetic definitions, this definition has the merit of being free from existential questions. If marks of a field (or entities of a range) exist, these elements exist. The element is a conception which is a function of its $n$ marks, or $n$ coördinates. The conception is one needed in many applications of mathematics, hence its introduction is justified. But logically these elements are not extensions of the ordinary numbers and can be so called only by an extension of the meaning of the term number, and this extension must be in an altogether artificial manner. As Bertrand Russell points out with regard to the elements defined in the next definition, we cannot identify * unity with any of the $n$-tuple elements, not even with the modulus, which may be written

$$
(1,0,0, \cdots, 0) \text {. }
$$

For unity is not $n$-tuple, not even an $n$-tuple with $n-1$ zero coördinates. The distinction is analogous to that which insists that unity and positive unity are two different conceptions.

The $n$-tuple has no reference to anything else. It is a compound conception built up directly from the range given. Its laws of combination are purely arbitrary, since there is no reason à priori why, in $(a, b)(x, y) \equiv\left(\gamma_{111} a x+\gamma_{121} a y+\gamma_{211} b x\right.$ $\left.+\gamma_{221} b y, \gamma_{112} a x+\gamma_{122} a y+\gamma_{212} b x+\gamma_{222} b y\right)$, the eight $\gamma$ 's should have one value rather than another. The thing to do, on this basis, obviously is to study all possible types of algebras of such $n$-tuples, and find their uses, if any.

\section{The Manifold.}

The second definition I shall call the Russell definition, although it has appeared before in works of other logicians. It runs thus :

What defines a hypercomplex number is a one-many relation whose domain consists of real numbers (that is, ordinary rationals, irrationals, etc.), and whose converse domain consists of the first $n$ integers (or, in the case of complex numbers of in-

\footnotetext{
* Principles of Mathematics, vol. 1, p. 380 .
} 
finite order, of all integers). These relations are indicated by $e_{1}, e_{2}, \cdots, e_{n}$. Thus, in $a=a_{1} e_{1}+a_{2} e_{2}+\cdots+a_{n} e_{n}, a_{1}$ is correlated to $1, a_{2}$ to $2, \cdots, a_{n}$ to $n$. The relation is one-many because $a_{i}$ may equal a given number $b$, for $i=1,2, \cdots, n$; that is, $b$ is correlated to each integer ; so for every number. These one-many relations may be defined to be the complex numbers. In this way a purely logical definition is obtained. We are not to identify $1 e_{1}+0 e_{2}+\cdots+0 e_{n}$ with $e_{1}$, that is, the complex number is a function of the coordinates, not of the relations $e$. Evidently we may use any other range than that of all real numbers, and this definition becomes as general as the first, which it most resembles. Russell proceeds immediately to show that this virtually defines a complex number to be an $n$-dimensional entity. It is substantially the definition at the foundation of vector analysis, where the relational symbols $e_{1}, e_{2}, \ldots, e_{n}$ are expressed by directions.

It is sufficiently clear that these manifolds are not in the ordinary number system any more than were the $n$ tuples.

'Their sums and their products are likewise manifolds whose coordinates are determined by certain laws of combination. True, we may call them numbers, but the identification of an ordinary number with any one of the manifolds is highly artificial. In fact, students of space analysis or vector analysis would scarcely conceive of any such identification being possible, any more than they would think of identifying an angle with its number of degrees.

The connection of the $n$-tuple and the manifold with the bilinear function of $n$ variables is evident.

This is the point of view of the qualitative-unit mathematician, a unit 1 correlated to the $n$ integers gives the $n$ units $1 e_{1}, 1 e_{2}, \cdots, 1 e_{n}$, of the system. These are usually written $e_{1}, \cdots, e_{n}$, although $1 e_{1}$ is not really $e_{1}$; for $e_{1}$ really expresses only that something is correlated to 1 . The correlation may as well be with $n$ directions in space, and might be written $i, j, k, \ldots$. In this case the qualitative phase is a little more apparent. Of course we are left free to say what $a e_{1} \times b e_{2}$ shall mean; this gives rise to a multiplication table, meaning that if $a$ is correlated to $1, b$ to 2 , then $a b$ shall be correlated to $\gamma_{121} e_{1}+\gamma_{122} e_{2}+\cdots+\gamma_{12 n} e_{n}$. This view appeals strongly to the quaternionist or vector analyst who desires a flexible symbolism, although many use the cumbrous $n$-tuple forms. 


\section{The Operator.}

The third definition I shall call the Peano definition, although it appeared long before in Hamilton's quaternions. This definition looks upon the hypercomplex number as an operator. What Peano* calls a complex number is incapable of multiplication, as he explicitly says, hence it is not the kind of entity we are discussing here. But he does not include quaternions, $\sqrt{-1}$, and the like, in the class of complex numbers. These he defines as operators. Thus, $\sqrt{-1}$ is the substitution $\left|\begin{array}{cc}0 & -1 \\ +1 & 0\end{array}\right|$, that is, if we have given a non-multiplicative manifold $(x, y)$, then by $\sqrt{-1}(x, y)$ we mean $(-y, x)$. Obviously matrices come under this head, as do linear substitutions.

It will readily be granted, I suppose, that these operators are not numbers, although Hamilton identifies quaternions that are purely scalars with the domain of positive and negative numbers. But he means that the scalar 1 is an operator such that applied to $a$, any vector, $1 a=a$. If we were to define numbers as operators, then, of course, scalars are numbers. But numbers defined on a pure arithmetical basis are not operators.

It is to be noted carefully in these three definitions that the product or sum of any two elements is a third element of the same character. No possible combination ever leads us away from the range under consideration. In the first case we have, for example, the products of two n-tuples: $(a, b)(x, y)=$ $\left(a x \gamma_{111}+a y \gamma_{112}+b x \gamma_{121}+b y \gamma_{122}, a x \gamma_{211}+a y \gamma_{212}+b x \gamma_{221}+\right.$ $\left.b y \gamma_{222}\right)$, an $n$-tuple. To connect these with numbers, we virtually must define real numbers to be $n$-tuples. In the second case we have for the product of two manifolds $\left(a e_{1}+b e_{2}\right)$ $\left(x e_{1}+y e_{2}\right)=\left[\left(a x \gamma_{111}+a y \gamma_{112}+b x \gamma_{121}+b y \gamma_{122}\right) e_{1}+\left(a x \gamma_{211}\right.\right.$ $\left.\left.+a y \gamma_{212}+b x \gamma_{221}+b y \gamma_{222}\right) e_{2}\right]$, a manifold. To connect with numbers, we must define numbers as manifolds. In the third case we have for the product of two operators $\left(a i_{1}+b i_{2}\right)$ $\left(x i_{1}+y i_{2}\right)()=\left[a x \gamma_{111}+a y \gamma_{112}+b x \gamma_{121}+b y \gamma_{122}\right) i_{1}+\left(a x \gamma_{211}\right.$ $\left.\left.+a y \gamma_{212}+b x \gamma_{221}+b y \gamma_{222}\right) i_{2}\right]$ ( ), an operator. To connect with numbers, we must define a number as an operator.

*Formulaire (1901). 


\section{The Congruence.}

The fourth definition I shall call the algebraic definition. It is so called by Russell, who says indeed regarding this method* ". . . the algebraic generalisation is very inferior to the arithmetical, since the latter gives all irrationals by a uniform method, whereas the former, strictly speaking, will give only the algebraic numbers. But with regard to the complex numbers, the matter is otherwise. No arithmetical problem leads to these, and they are wholly incapable of arithmetical definition. But the attempt to solve such equations as $x^{2}+1=$ $0, x^{2}+x+1=0$ at once demands a new class of numbers, since, in the whole domain of real numbers, none can be found to satisfy these equations. To meet such cases, the algebraic generalisation defined new numbers by means of the equations whose roots they were. . . But the algebraic generalisation was wholly unable (as it was, in truth, at every previous stage) to prove that there are such entities as those which it postulated."

Without agreeing or disagreeing with this statement as it stands, we may place the algebraic definition on the following unimpeachable basis : $\dagger$ Let us consider that $i$ is such an entity that wherever we find the expression $i^{2}+1$ we may write 0 for it ; that is, we reduce all our problems, as it were, modulo $i^{2}+1$. Then we may write $x^{2}+x+1=x^{2}+x+\frac{1}{4}+\frac{3}{4}=$ $x^{2}+x+\frac{1}{4}+\frac{3}{4}-\frac{3}{4}\left(i^{2}+1\right)=x^{2}+x+\frac{1}{4}-\frac{3}{4} i^{2}$. This evidently has the factors $x+\frac{1}{2}-\frac{1}{2} v^{\prime} \overline{3}$ and $x+\frac{1}{2}+\frac{1}{2} \sqrt{3} i$. Hence we may speak of the roots as $-\frac{1}{2}+\frac{1}{2} \sqrt{3} i$ and $-\frac{1}{2}-$ $\frac{1}{2} \sqrt{3} i$. We have not made any assertions at all regarding the nature of $i$, and may if we choose look upon it as simply a perfectly arbitrary number, or algebraic form destitute of numerical value. We may look upon all radicals in the same way; thus $x^{2}+6 x+7$ has no rational roots. But if we work modulo $j^{2}-2$, we find at once that we may write $x^{2}+6 x+7$ $=x^{2}+6 x+9-j^{2}$, and we say $x+3 \pm j=0$. We call $j$ an extension of our number system. So we call $i$ an extension of the number system.

\footnotetext{
* Principles of Mathematics, p. 377 et seq.

$\dagger$ With regard to this basis, I desire to acknowledge valuable suggestion from Professors Moore and Dickson.
} 
If now we go farther and work modulo $i^{2}+1, j^{2}+1, k^{2}+1$, $l^{2}+1, \ldots$, where $i, j, k, l, \ldots$ are different forms, we find that we may take $(i+j)^{2}+2=0$ if $i j+j i=0$. Hence it is suggested that we must either consider $i j=0$, or else consider that $i j$ and $j i$ are not equal. That is, we must choose between nilfactors with commutativity, or else give up commutativity. We do the latter and arrive at quaternions. So that we have expressions reducible modulo

$$
\begin{array}{cccc}
i_{1}^{2}+1, \quad j_{1}^{2}+1, \quad k_{1}^{2}+1, & i_{2}^{2}+1, \quad j_{2}^{2}+1, \quad k_{2}^{2}+1, \ldots, \\
i_{1} j_{1}+j_{1} i_{1}, \quad j_{1} k_{1}+k_{1} j_{1}, & k_{1} i_{1}+i_{1} k_{1}, \quad i_{2} j_{2}+j_{2} i_{2}, \ldots, & \\
i_{1} j_{2}, \quad i_{2} j_{1}, \quad i_{1} k_{2}, & i_{2} k_{1}, \quad j_{1} k_{2}, & j_{2} k_{1}, \quad j_{1} i_{2}, \quad j_{2} i_{1}, \\
k_{1} i_{2}, \quad k_{2} i_{1}, \quad k_{1} j_{2}, \quad k_{2} j_{1}, & \cdots
\end{array}
$$

If we use only $i_{1}, j_{1}, k_{1}$, we find we may factor the expression

namely

$$
x^{2}-2 a x+a^{2}+b^{2}+c^{2}+d^{2},
$$

$$
\begin{aligned}
x^{2}-2 a x+a^{2}+b^{2}+c^{2}+d^{2}=\left(x-a+b i_{1}+c j_{1}+d k_{1}\right) \\
\left(x-a-b i_{1}-c j_{1}-d k_{1}\right) .
\end{aligned}
$$

We have thus arrived at quaternions. Here again we may consider $i_{1}, j_{1}, k_{1}$ as merely formal, or we may look on them as extensions of the number system. In the end the latter view seems more profitable.

We may then state the algebraic definition for the general case as follows: Let there be an expression, or set of expressions, in terms of $q, r, \cdots$ such that we reduce all expressions in terms of the entities of a given range, modulo these given expressions, where $q, r, \ldots$ represent any entities for which this can be done. Then the entities $q$, say $q_{1}, q_{2}, \ldots$, and the entities $r$, say $r_{1}, r_{2}, \cdots$, etc., define extensions of the given range. If the number of these ideal entities is finite we arrive at an algebra of a finite number of dimensions, otherwise an algebra of infinite dimensions.

\section{Conclusions.}

This brings us to one of the objects of the whole discussion. Let me put it in the form of a simple question. Do positive and negative numbers constitute a complex number system of two units $e_{0}=+1, e_{1}=-1$, with the domain of ordinary 
arithmetical rational and irrational numbers for the coordinate range, or do they not constitute a complex system, and, if not, why not? Surely +1 and -1 are units and are qualitatively distinct. According to the second definition, then, we may define $a_{1}(+1)+a_{2}(-1)$ as a complex number of two units. But in this algebra we know that $a_{1}(+1)+a_{2}(-1)=\left(a_{1}-\right.$ $\left.a_{2}\right)(+1)$ when $a_{1}>a_{2} ;=0$, when $a_{1}=a_{2} ;$ and $=\left(a_{2}-a_{1}\right)(-1)$ when $a_{2}>a_{1}$. Hence these qualitative units are such that though themselves distinct, when coordinates are attached, we arrive at expressions which are not unique. Thus if $\left(a_{1}, a_{2}\right)=$ $\left(b_{1}, b_{2}\right)$, we may infer $a_{1}=b_{1}, a_{2}=b_{2}$; and if $\left(a_{1} e_{1}+a_{2} e_{2}\right)=\left(b_{1} e_{1}\right.$ $\left.+b_{2} e_{2}\right)$, then $a_{1}=b_{1}, a_{2}=b_{2}$. But if $a_{1}(+1)+a_{2}(-1)=$ $b_{1}(+1)+b_{2}(-1)$, the most we can infer is that $a_{1}=b_{1} \pm b, a_{2}=$ $b_{2} \pm b$. We meet the same problem in operators and simply evade it by defining $(+1) \alpha+(-1) \alpha=0 \alpha$. The question still remains.

If an $n$-tuple may reduce to an $(n-m)$-tuple it ceases to be properly an $n$-tuple. Hence the $n$-tuple algebra must exclude the positive-and-negative-number calculus as not an algebra. So also if the $n$-manifold may have its number of dimensions reduced, it ceases to be an $n$-manifold. So this calculus must be excluded here. A vector algebra of positive and negative quantities is one-dimensional and not properly a vector algebra at all. In fact, the $n$-tuple and the manifold exclude any possibility of ever reducing the number of positions or of units by additions of their coordinates which belong to different positions or different units. Technically, the units are linearly independent so far as entities of the range are concerned. Now $+1,-1$, are not linearly independent so far as the domain of the integer or the real number are concerned. Thus, for example $2(+1)+1(-1)=1(+1)$.

The third definition places the difficulty farther back. Thus $(+1)$ and $(-1)$ are independent or not according as $(+1) a$ and $(-1) a$, where $a$ is the operand, are independent or not.

The fourth definition admits such lack of independence, for we define -1 by this very lack of independence. Indeed, we define -1 to be the entity $x$ such that

$$
x+1=0 \quad \text { or } \quad x+a=a-1 .
$$

That is, we work modulo $x+1$. We extend our old arithmetical addition to a new ideal range. On the basis of the 
fourth definition, positive and negative numbers do constitute an algebra of two distinct qualitative units, but not a two-dimensional algebra. In fact, we may assert at once that the fourth definition defines ideal numbers which are qualitatively distinct, but which may not be able to be ordered as many-dimensional. We do not at first know whether the different ideals are many-dimensional or not. Thus $q^{2}-2 w q+t^{2}=0$ is satisfied by any number of sets of quaternions, and the dimensions may thus become infinite.

The distinction may be illustrated by the difference between a line in a two-dimensional bounded non-euclidean space and such space itself. The portion of the line external to the space may be called ideal, but the real and the ideal (qualitatively different) portions of the line are yet one-dimensional.

Modifications of the usual theory of algebras are necessary in these algebras with, we may say, deficiency of dimension. Thus for positive and negative numbers we do not have a characteristic equation of order two, which we should expect, but simply the equation

$$
(+1)+(-1)=0 \text {. }
$$

Another example occurs in ordinary complex numbers, which constitute an algebra of four qualitative units, $e_{0}, e_{1}, e_{1}^{2}, e_{1}^{3}$, with the equations $e_{0}+e_{1}^{2}=0=e_{1}+e_{1}^{3}$. The characteristic equation is not of order four, but for $\rho=x e_{0}+y e_{1}+z e_{1}^{2}+w e_{1}^{3}$ is $(\rho-x+z)^{2}+(y-w)^{2}=0$.

As another example we might define an algebra by working modulo $e_{1}^{2}-1, e_{2}^{3}-1, e_{1} e_{2}-e_{2}^{2} e_{1}, e_{2}+e_{2}^{2}+1, e_{1} e_{2}+e_{1} e_{2}^{2}+e_{1}$.

These examples show the great power and generality of the fourth definition. It is evident that we define an algebra thus by what may be called its invariant equations.

The criticism made by Russell, that algebraic definitions do not guarantee the existence of the thing defined, does not apply to this method of putting the definition. If algebra exists, then we may work modulo $f(q)$ if we choose, and $f(q)$ is simply an expression in the algebra. We thus arrive at expressions containing $q$. If we choose to call these hypercomplex numbers, we do not thereby destroy them. If under given conditions it turns out that we may work modulo a whole infinity of expressions $f\left(q_{1}\right), f\left(q_{2}\right), \ldots$, then we have simply found a kingdom where we searched for much less.

Aprl, 1906. 